\title{
Development and characterisation of a 3D multi-cellular in vitro model of normal human breast: a tool for cancer initiation studies
}

\author{
Claire E. Nash ${ }^{1,5}$, Georgia Mavria ${ }^{1}$, Euan W. Baxter ${ }^{1}$, Deborah L. Holliday ${ }^{1}$, Darren \\ C. Tomlinson ${ }^{2}$, Darren Treanor ${ }^{1,3}$, Vera Novitskaya ${ }^{4}$, Fedor Berditchevski ${ }^{4}$, Andrew \\ M. Hanby ${ }^{1}$ and Valerie Speirs ${ }^{1}$ \\ ${ }^{1}$ Leeds Institute of Cancer and Pathology, University of Leeds, Leeds, UK \\ ${ }^{2}$ Leeds Institute of Biomedical and Clinical Sciences, University of Leeds, Leeds, UK \\ ${ }^{3}$ Leeds Teaching Hospitals NHS Trust, Leeds, UK \\ ${ }^{4}$ School of Cancer Sciences, University of Birmingham, Birmingham, UK \\ ${ }^{5}$ Current address: The Research Institute of the McGill University Health Centre, Decarie Boulevard, Montreal, Quebec, \\ Canada
}

Correspondence to: Valerie Speirs, email: v.speirs@leeds.ac.uk

Keywords: 3D cell culture, breast, HER2

Received: January 09, $2015 \quad$ Accepted: March 18, $2015 \quad$ Published: April 12, 2015

This is an open-access article distributed under the terms of the Creative Commons Attribution License, which permits unrestricted use, distribution, and reproduction in any medium, provided the original author and source are credited.

\section{ABSTRACT}

Multicellular 3-dimensional (3D) in vitro models of normal human breast tissue to study cancer initiation are required. We present a model incorporating three of the major functional cell types of breast, detail the phenotype and document our breast cancer initiation studies. Myoepithelial cells and fibroblasts were isolated and immortalised from breast reduction mammoplasty samples. Tri-cultures containing non-tumorigenic luminal epithelial cells HB2, or HB2 overexpressing different HER proteins, together with myoepithelial cells and fibroblasts were established in collagen I. Phenotype was assessed morphologically and immunohistochemically and compared to normal breast tissue. When all three cell types were present, polarised epithelial structures with lumens and basement membrane production were observed, akin to normal human breast tissue. Overexpression of HER2 or HER2/3 caused a significant increase in size, while HER2 overexpression resulted in development of a DCIS-like phenotype. In summary, we have developed a 3D tri-cellular model of normal human breast, amenable to comparative analysis after genetic manipulation and with potential to dissect the mechanisms behind the early stages of breast cancer initiation.

\section{INTRODUCTION}

Researchers are beginning to appreciate the need for more complex multicellular three-dimensional (3D) laboratory models in order to study the processes associated with disease progression. In recent years there has been a shift towards developing $3 \mathrm{D}$ in vitro models that better represent breast tissues. These typically involve culturing breast epithelial cells alone or with fibroblasts [1-3] in 3D in the presence of extracellular matrix (ECM) such as Matrigel ${ }^{\mathrm{TM}}$ or collagen I. Mammary luminal epithelial cells respond to ECM by forming acini-like structures [4]. By culturing cells with specific ECM constituents, cell-cell and cell-ECM interactions can be represented in the laboratory [5]. This has led to a better understanding of how different breast cell types interact with each other and the surrounding ECM as well as identification of key intercellular signalling proteins involved in maintaining luminal cell polarity such as integrins [6] and cadherins [7].

Current $3 \mathrm{D}$ in vitro models of normal human breast are limited. Compared to the wealth of breast cancer cell lines available to represent the molecular subtypes of breast cancer [8], normal breast epithelial cell lines are 
scarce. MCF10A is the most commonly used cell line to represent normal breast. When cultured in Matrigel $^{\mathrm{TM}}$, polarised acini-like structures with hollow lumens develop which produce basement membrane proteins $[9,10]$. These models often incorporate fibroblasts [13]. However, MCF10A cells have a propensity to adopt a basal phenotype $[11,12]$ and are sensitive to changes in culture conditions [13] making them challenging to work with. Furthermore there are limitations to using Matrigel $^{\mathrm{TM}}$; although used widely, the levels of growth factors and basement membrane components are heterogeneous [14] and the substance itself is prepared from Engelbreth-Holm-Swarm mouse sarcoma cells. The main constituent of breast stroma is collagen I [15], a regulator of morphology and phenotype of breast epithelial cells $[1,16,17]$. Moreover, variation in the amount of collagen and subsequent mammographic density may be linked to the onset of breast cancer $[18,19]$. Thus the case for incorporating collagen I in the development of 3D in vitro models of breast tissue is strong.

In addition to the role fibroblasts play on regulating luminal epithelial behaviour [20-22], breast myoepithelial cells are important in maintaining luminal epithelial polarisation and architecture [23, 24]. These are considered tumour suppressive $[25,26]$, expressing a number of tumour suppressor proteins e.g. maspin and ER $\beta$, structural ECM proteins e.g. fibronectin, proteinase inhibitors e.g. tissue inhibitor of metalloproteinase-1 (TIMP1) and angiogenic inhibitors e.g. thrombospondin-1 $[25,27,28]$. However, studies incorporating these cells into 3D models are limited.

This study aimed to create in vitro a $3 \mathrm{D}$ tri-culture system of normal human breast that incorporates an alternative normal luminal epithelial cell line HB2 [29, 30], together with myoepithelial and fibroblast cells in a physiologically relevant matrix, collagen I. We demonstrated that the model is representative of normal human breast by comparing the morphology and immunoprofile to 'gold standard' normal human breast tissue sections, and determined that the model was amenable to cancer initiation studies through HER overexpression in luminal epithelial cells.

\section{RESULTS}

\section{D in vitro tri-culture model recapitulates the morphology and phenotype of normal human breast tissue}

HB2 cells were cultured alone, with Myo1089 cells or with Myo1089 and fibroblasts in collagen I gels and phenotype assessed by H\&E after 21 days. When cultured alone, HB2 cells formed tight spherical units of varying size with occasional scattered cells (Figure 1a, 1b). A similar phenotype was observed when HB2 were co-cultured with Myo1089 cells (Figure 1c, 1d). Tri-culture of HB2, Myo1089 and fibroblasts, resulted in the arrangement of Myo1089 myoepithelial cells around the outer edges of spherical lumen containing HB2 epithelial cells with fibroblasts loosely distributed throughout the collagen gel (Figure 1e) akin to normal breast acini (Figure 1f). This phenotype was only observed when all 3 cell types were present. Spatial localisation of myoepithelial cells and fibroblasts were confirmed by immunohistochemistry (Figure 1g, 1h), respectively.

We then conducted immunohistochemical characterisation of $3 \mathrm{D}$ in vitro tri-cultures and compared this to normal human breast tissue. As shown in Figure 2, tri-culture phenotype (a-d, i-l) was strikingly similar to that of normal breast tissue (e-h, m-p). Controls (no primary antibody) are shown in Figure $2 \mathrm{a}$ and $2 \mathrm{i}$. E-cadherin (E-cad) was expressed at cell-cell junctions (Figure 2b, 2f) with EMA at apical membranes (Figure 2c, 2g). Some cell proliferation was identified by Ki67 staining (Figure 2d, 2h), with apoptosis detected within central lumens by M30 staining (Figure 2i, 2m). $\alpha$-SMA was specific to myoepithelial cells (Figure $2 \mathrm{j}, 2 \mathrm{n}$ ) while basement membrane production was detected around outer edges of breast ducts by collagen IV expression (Figure 2k, 2o). Vimentin expression was detected in both myoepithelial cells and fibroblasts (Figure 21, 2p). Comparison of these immunohistochemical features to normal breast tissue sections demonstrated strikingly strong similarities.

\section{Overexpression of HER proteins affect the morphology and phenotype of the tri-culture model}

To test whether the tri-culture model was suitable for cancer initiation studies, HER2 and HER3 proteins were overexpressed in HB2 cells. Protein presence was confirmed by western blot analysis (Figure 3a). Compared to controls (Figure 3b) upon HER2 overexpression, HB2 cells formed larger, elongated units with distorted lumens and the appearance of protrusions from outer edges (Figure 3c). HER3 overexpressing cells also formed larger units containing distorted lumens but retained a spherical shape (Figure 3d). HB2 cells overexpressing both HER2 and HER3 formed a combination of smaller rounded units and larger elongated units that were larger than HB2 controls and also featured distorted lumens (Figure 3e). Quantitative analysis supported these observations. HB2 co-units significantly increased in size following HER2, or HER2/3 overexpression (Figure 3f, $p=<0.05$ ). Overexpression of HER3 induced a significant decrease (Figure $3 \mathrm{~g}, p=0.0442$ ) in co-unit formation, while overexpression of HER2, HER3 and HER2/3 caused a decrease in lumen formation compared to HB2 controls (Figure $3 \mathrm{~h}, p=<0.05$ ). To examine if all 3 cell types 


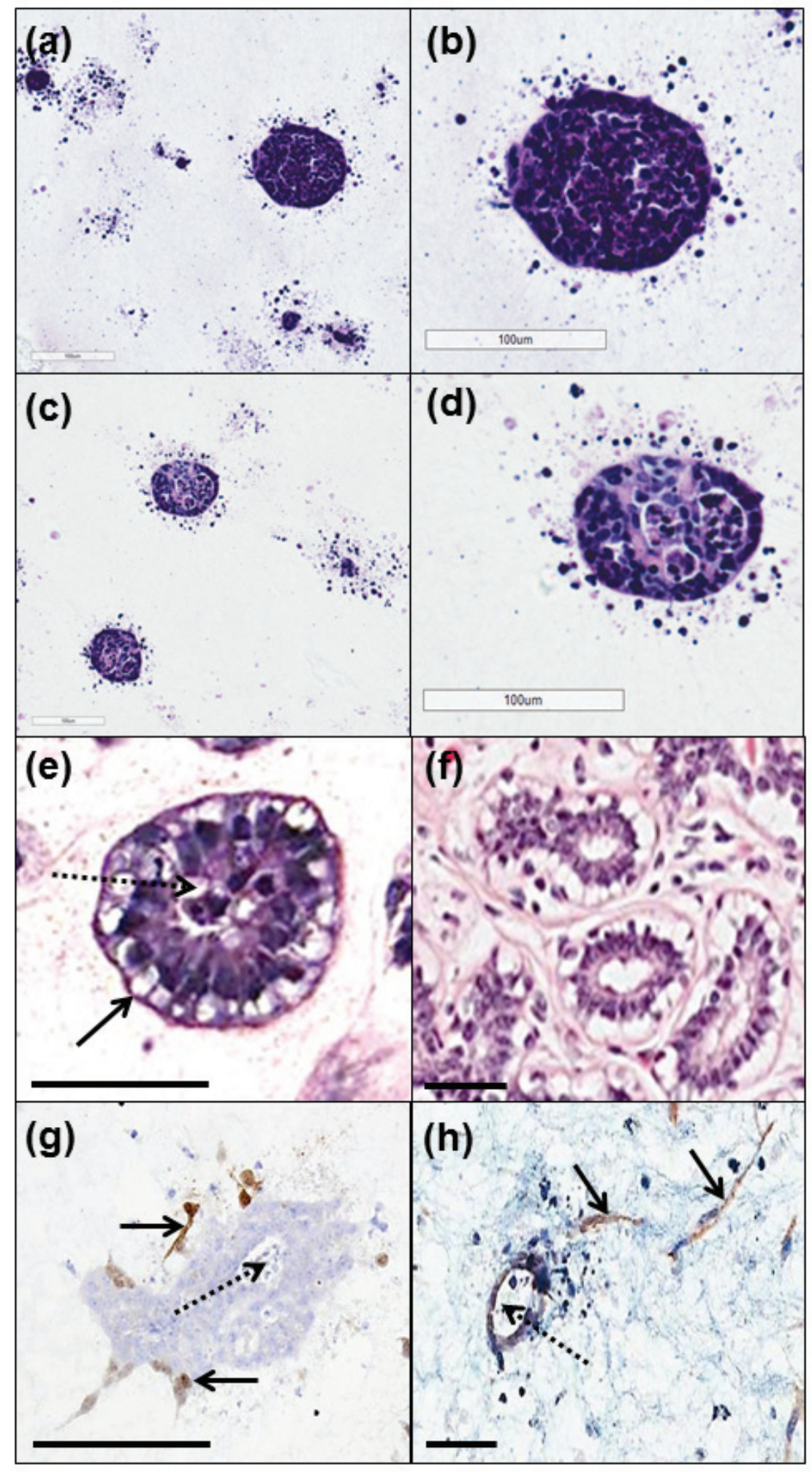

Figure 1: Comparison of morphology of 3D tri-culture model with normal human breast tissue. When cultured alone $(\mathbf{a}, \mathbf{b})$, HB2 cells formed tight spherical units of varying size with occasional scattered cells. A similar phenotype was observed when HB2 were co-cultured with Myo1089 cells (c, d). In tri-cultures containing HB2, Myo1089 and fibroblasts, ductal structures with hollow lumens (dotted arrow) were observed surrounded by a border of clear myoepithelial cells (arrow; e) akin to normal human breast acini (f). Immunohistochemical characterisation using tGFP and dsRed antibodies confirmed the distribution of myoepithelial cells at the outer edges of HB2 structures with hollow lumens (dotted arrow) (arrows; g) and fibroblasts loosely distributed throughout collagen gels (arrows; h), respectively. Scale bars $=100 \mu \mathrm{m}$ 
were necessary to mediate these phenotypic changes, HER overexpressing HB2 cells were co-cultured with either myoepithelial cells or fibroblasts. Co-culture with myoepithelial cells had an organisational effect inducing the formation of rounded cohesive units while co-culture with fibroblasts had the opposite effects, resulting in larger co-units with a discohesive and disorganised appearance (Suppl. Figure 3).

\section{HER2 overexpressing HB2 3D tri-cultures resemble human DCIS phenotype}

H\&E stained sections of HER2 overexpressing and HB2 control 3D tri-cultures were compared with a cross section of a human breast tissue that contained areas of both DCIS and normal adjacent acini (Figure 4a). As previously, HB2 control units were small, rounded, contained lumens (Figure $4 \mathrm{~b}$ ) and were similar phenotypically to normal breast acini (Figure 4a). In the breast tissue section, areas of DCIS featured large elongated ducts and contained distorted lumens with necrotic tissue (Figure 4a). These also contained enlarged and discohesive cells. HER2 overexpressing HB2 units were similarly elongated in shape, containing discohesive cells with distorted luminal spaces thought to be a product of necrosis (Figure 4c). Immunohistochemical characterisation showed no change in Ki67 expression between HB2 control units or those containing HER2overexpressing cells, however a reduction in M30 and E-cadherin was observed (Suppl. Figure 4), suggesting these morphological changes are a result of apoptosis and loss of cell adhesion. This not only confirmed that the normal 3D tri-culture model was robust and retained features of normal breast acini, but also that morphological and phenotypical features of DCIS could be recapitulated through overexpression of HER2 in the 3D tri-culture model.

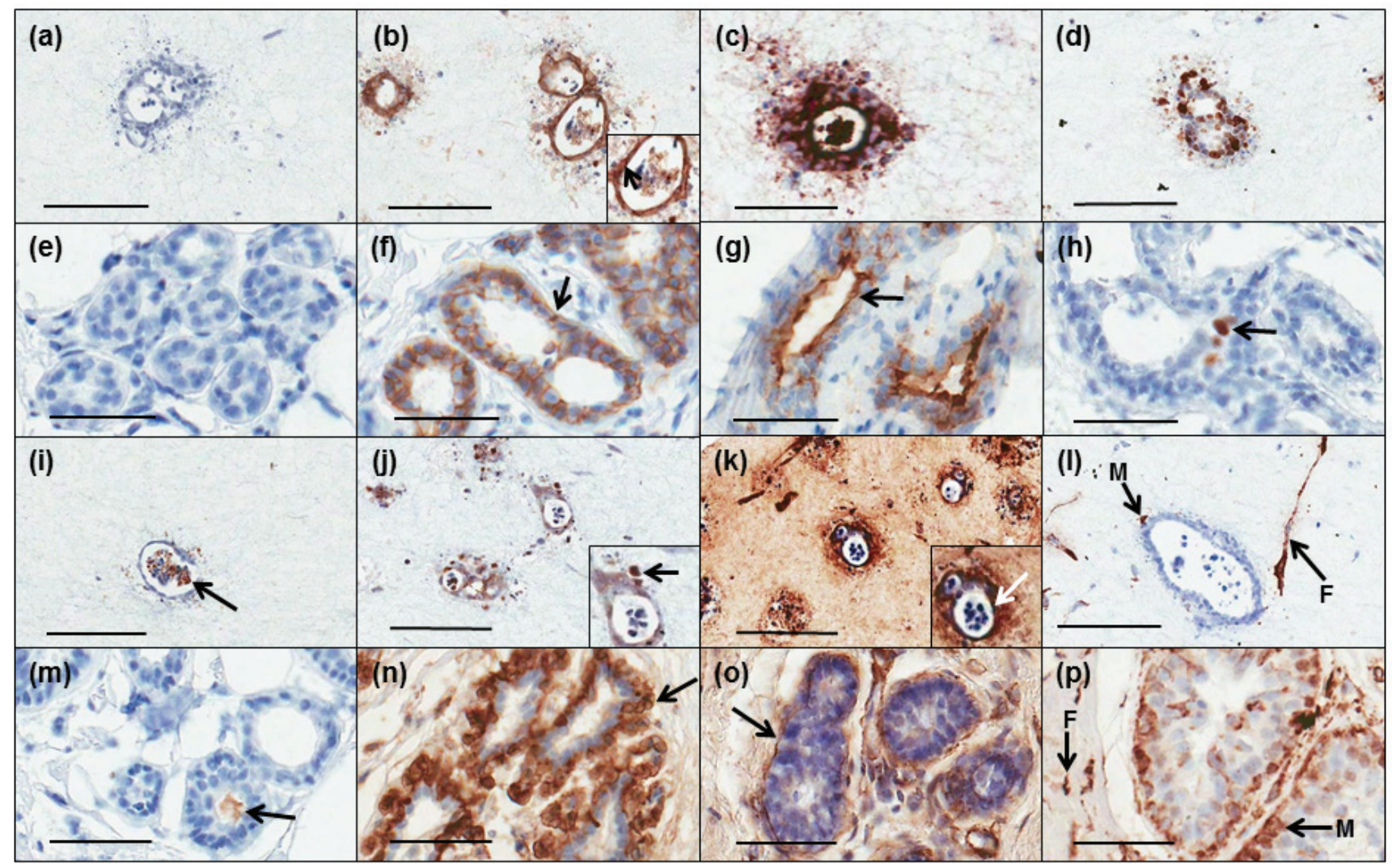

Figure 2: Comparison of immunohistochemical profiles of 3D tri-cultures with those of normal breast tissue. The phenotype of 3D in vitro tri-cultures (a-d; i-l) was assessed by immunohistochemistry and compared to cross sections of breast reduction mammoplasty tissue (e-h; m-p). Negative controls (a, e). E-cadherin was expressed by HB2 epithelial cells at cell-cell junctions (b, arrow) similar to luminal epithelium of breast acini (f, arrow). EMA expression was concentrated at the HB2 cell-lumen interface in co-units (c) and was specific to apical membranes of luminal epithelium of breast acini (g, arrow).The majority of cells within HB2 co-units expressed Ki67 (d) with scattered positivity within breast acini (h). M30 staining demonstrated apoptosis within lumens of HB2 co-units (i, arrow) and also in breast acini ( $\mathrm{m}$, arrow). $\alpha$-SMA was restricted to myoepithelial cells on the periphery of HB2 co-units ( $\mathrm{j}$, arrow) while a continuous layer of $\alpha$-SMA positive myoepithelial cells surround luminal epithelium of breast acini (n, arrow).Collagen IV was concentrated around outside edges of HB2 co-units (k, arrow) and similarly is demonstrated encapsulating breast acini (o, arrow). Vimentin was expressed in both Myo1089 cells (M) and fibroblasts (F) in the tri-culture model (I) analogous to Vimentin positivity in myoepithelial cells (M) and fibroblasts $(F)$ in normal breast tissue $(\mathbf{p})$. Original magnification for tri-culture model $=20 x$; for normal tissue $=40 x$. 
(a)

HB2

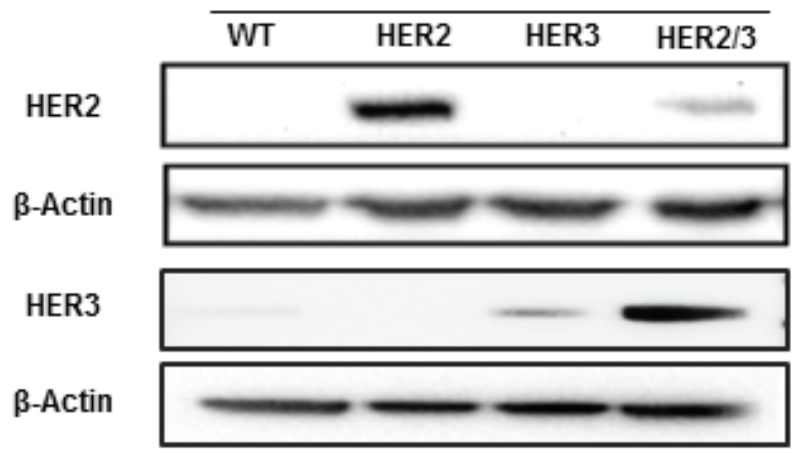

(b)

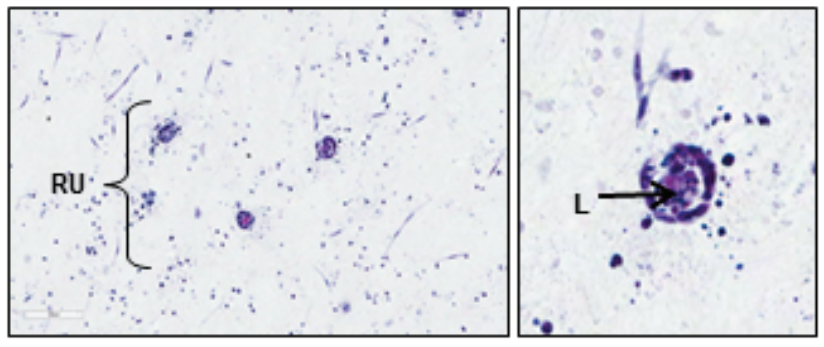

(c)
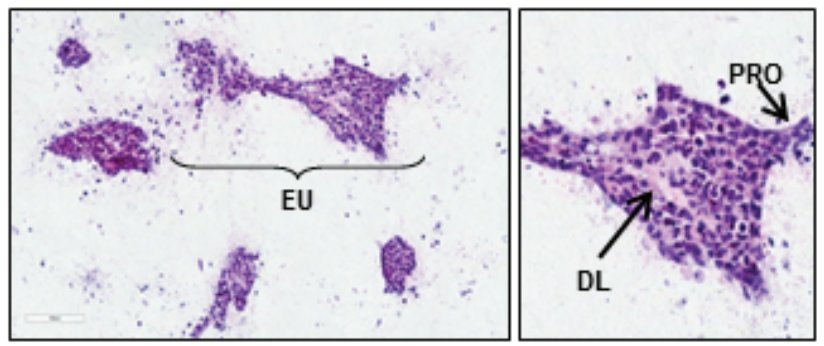

(d)


(e)

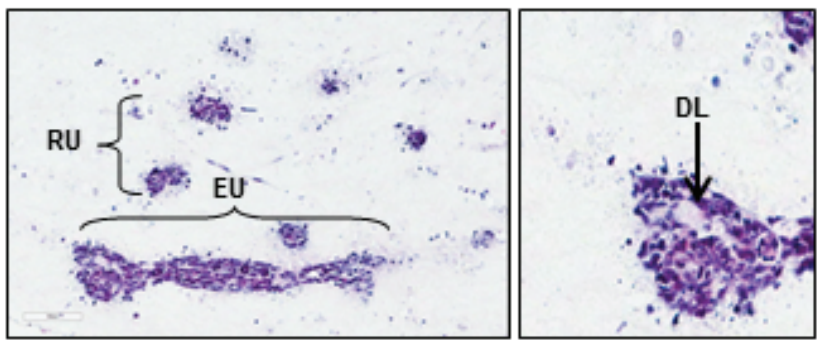

(f)



(g)

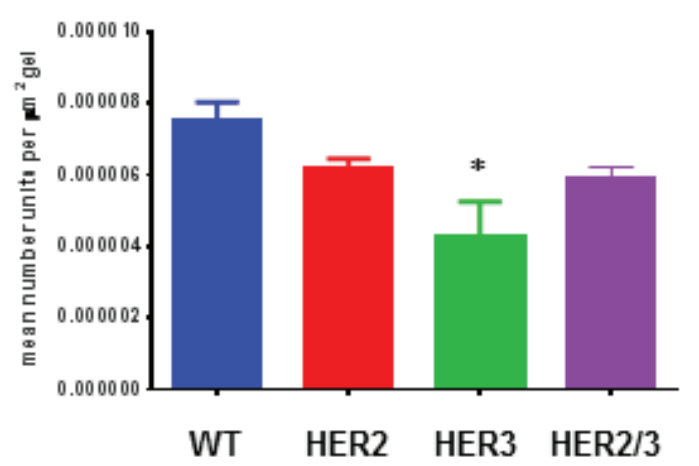

(h)



Figure 3: Overexpression of HER proteins in HB2 cells affects 3D architecture. Stable overexpression of HER2 and HER3 in HB2 cells was shown by western blot (a). WT cells in 3D tri-culture (b) formed rounded units (RU) with lumens (L). When HER2 was overexpressed (c), formation of much larger elongated units (EU) with distorted lumens (DL) and protrusions (PRO) from edges was observed. HER3 overexpression (d) resulted in rounded units with distorted lumens (DL) while overexpression of HER2 and HER3 cells (e) resulted in a combination of rounded units (RU) and elongated units (EU) with distorted lumens (DL). Quantitative analysis confirmed HER2 and HER2/3 overexpression induced significantly larger HB2 unit formation (f). Only HER3 overexpression alone significantly altered the number of HB2 units formed (g) while HER2, HER3 and HER2/3 overexpression significantly reduced lumen formation (h). Bars denote mean from three gel replicates and error bars standard error of the mean (SEM). $*=p<0.05$ 


\section{DISCUSSION}

With scientists now recognising the importance of the microenvironment in cancer initiation and progression, there is an increased need for robust $3 \mathrm{D}$ in vitro models which incorporate components of the microenvironment. Here, we present, a 3D in vitro model of normal human breast that incorporates not only breast luminal epithelium with fibroblasts, but also myoepithelial cells all embedded in the physiologically relevant matrix collagen I. This model reflects the morphology and phenotype of normal breast providing a novel tool to investigate the mechanisms behind the early events of cancer initiation and the formation of pre-malignant lesions.

Following optimisation of culture conditions including culture medium, cell numbers and duration of culture (data not shown), we demonstrated that coculturing the three major cell types comprising the mammary duct in collagen I resulted in the arrangement of a border of myoepithelial cells surrounding HB2 epithelial cells which formed ductal structures with hollow lumens, with fibroblasts loosely distributed throughout the collagen gel. This morphology was remarkably similar to 'gold standard' normal human breast acini. It was necessary to use HB2 cells instead of the more commonly used MCF10A cells as we found that, as others have reported $[11,12]$, the latter exhibited plasticity, with evidence of at least 2 different sub-populations particularly when these were incorporated into more complex multicellular 3D collagen I models (Nash et al., manuscript in preparation).

This was confirmed further by immunohistochemical analysis of a panel of breast epithelial and mesenchymal markers and expression profiles compared to normal breast tissue. Near-identical expression patterns provided confidence that our $3 \mathrm{D}$ in vitro tri-culture model was a robust model of normal human breast tissue. Deposition of basement membrane protein collagen IV around the outer edges of co-units was observed, supporting previous reports that inclusion of myoepithelial cells with luminal epithelial cells in 3D collagen I matrix can abrogate
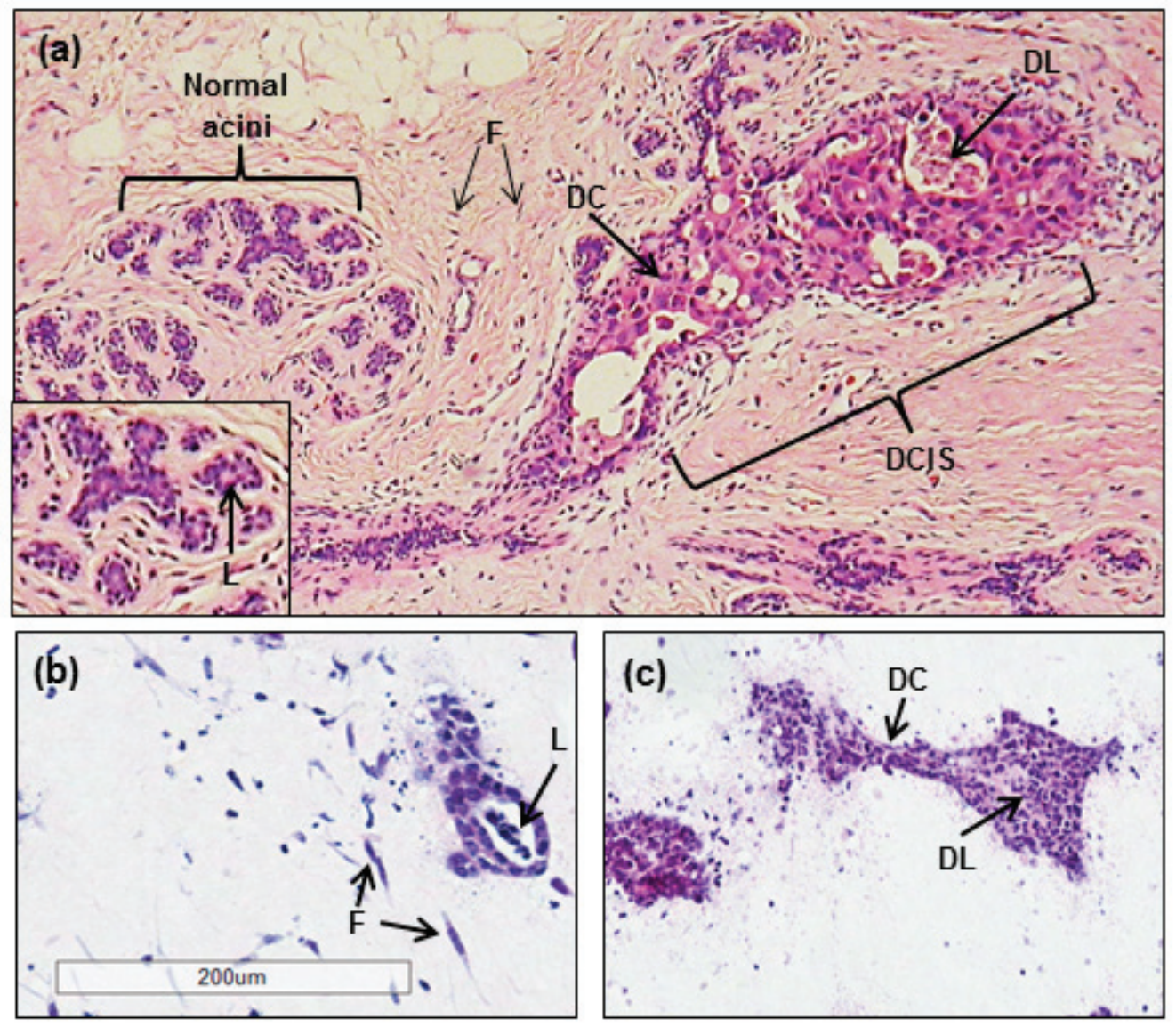

Figure 4: Comparison of HER2 overexpressing tri-cultures to human breast tissue containing DCIS. A cross section of human breast tissue (a) shows normal acini which are small and rounded with cleared central lumens (L, inset). Areas of DCIS consist of elongated discohesive cells (DC) with distorted lumens containing necrotic cells (DL). Co-units of the tri-culture model are also small and rounded with central lumens (L), strongly resembling normal breast acini. Overexpression of HER2 in luminal epithelium (c) resulted in an elongated, branched shape, the appearance of distorted lumen with possible necrosis (DL) and also discohesive cells (DC), reminiscent of a DCIS-like phenotype. Original magnification x20 (tri-culture model); 40x (breast tissue). 
the need for basement membrane preparations such as Matrigel $^{\mathrm{TM}}$ [24] due to their ability to produce proteins required for basement membrane formation [25]. All co-units displayed E-cadherin expression between HB2 cell junctions with epithelial membrane antigen situated at the apical-luminal interface of HB2 cells indicating the polarisation of HB2 cells. The presence of lumens cleared via apoptosis as detected by M30 expression. In addition, we proved that each cell type retained a phenotype representative of their corresponding cell type in normal breast tissue with vimentin expression detected in Myo1089 and fibroblasts and $\alpha$-smooth muscle actin found in Myo1089 cells only. Continued correlation to normal human breast tissue supports the validity of the tri-cultures as models of normal breast acini.

We then sought to determine if the model could be used as a tool to study cancer initiation processes. Focussing on the luminal epithelium, we aimed to disrupt the normal architecture to produce a morphology and phenotype akin to early pre-malignant breast lesions, through overexpression of HER proteins. HER2 gene amplification and protein overexpression is observed in around $16 \%$ of breast cancers [31] and is associated with a poor prognosis and survival in breast cancer patients due to its relationship with more aggressive tumours [32]. HER2 overexpression also occurs in up to $50 \%$ of DCIS [33] suggesting a role in cancer initiation with murine studies demonstrating HER2 overexpression alone is sufficient to induce transformation of mammary epithelium in a single step [34, 35]. This demonstrates the potency of HER2 as an oncogene. The HER2-HER3 heterodimer is the most potent and mitogenic heterodimer of the HER family. The potential of the HER2-HER3 heterodimer to activate PI3K [36] and the MAPK pathway [37, 38] makes HER2 a key regulator of a variety of cell responses such as proliferation, apoptosis and differentiation. Our study demonstrated that overexpression of HER2 alone had the most dramatic effect on the architecture of counits. These were significantly larger, more elongated and less cohesive, and featured protrusions similar to DCIS. Furthermore, lumen formation was significantly reduced within these co-units. Immunohistochemical characterisation suggested that these features may be attributed to apoptosis and a reduction in cell adhesion. Previous reports have proposed that HER2 overexpression in MCF10A cells in 3D Matrigel ${ }^{\mathrm{TM}}$ can result in evasion of apoptosis, lumen filling, disengagement of inner cell layers from the basement membrane, an increase in epithelialmesenchymal transition and cell migration, and that these can be attributed to the cooperation of EGF signalling with the TGF- $\beta$ signalling pathway and alteration of integrin signalling [39-41].

Despite reports implicating the HER2-HER3 heterodimer as the most potent and mitogenic dimer pair [36-38], in our model, HER3 or HER2/3 overexpression had a less pronounced effect on epithelial architecture, suggesting that the HER2-HER3 heterodimer was not the most potent inducer of aberrant epithelial architecture. Previous reports have demonstrated that the HER2-EGFR heterodimer is also strongly mitogenic and can drive cell migration $[42,43]$.

The effect of HER overexpression on luminal epithelial architecture has not been studied previously in the presence of both myoepithelial cells and fibroblasts. Here, we illustrated that myoepithelial cells in co-culture with HB2 cells appeared to diminish the disruptive effects of HER overexpression, akin to their recognised tumour suppressive role. On the other hand, co-culture with fibroblasts appeared to promote the opposite effect.

In summary we have successfully produced a robust heterotypic $3 \mathrm{D}$ in vitro model of normal human breast tissue that incorporated the three major cell types found in the breast in a physiologically relevant matrix of collagen I. We demonstrated individual cell populations were traceable, and formed co-units which shared morphology and phenotype with breast acini of the normal human mammary gland. Phenotypically, HER2 overexpressing tri-cultures had a strikingly similar phenotype to DCIS. Features such as elongated shape, distorted lumens, the presence of discohesive cells and necrosis were all recapitulated upon overexpression of HER2 in the model. The ability to produce structures that reflect early premalignant lesions such as DCIS in a physiologically relevant context validated the suitability of our model for cancer initiation studies. The model is robust, amenable to genetic manipulation and can be analysed and quantified by standard laboratory techniques. Our in vitro model could be used to dissect the mechanisms behind the early stages of breast cancer initiation.

\section{MATERIALS AND METHODS}

\section{Tissue}

Reduction mammoplasty samples were collected with informed consent via the Leeds Breast Cancer Campaign Tissue Bank following ethical approval (REC: 09/H1306/108, 10/H0308/48).

\section{Cell culture}

The non-tumorigenic breast epithelial cell line, HB2, was used [44]. The myoepithelial cell line Myo1089 was derived from breast reduction mammoplasty tissue and transduced with SV40 large T antigen [2] (gift from Professor Mike O'Hare, Ludwig Institute, London, UK). HB2 cells overexpressing HER2 and HER3 were generated through transfection of pSV2-HER2 [45] or retroviral transduction of pLXSN-ErbB3 [46], followed by single-stain or double-stain fluorescence activated 
cell sorting (FACS) for HER2 and HER3 or HER $2 / 3$ overexpressing cells, respectively to isolate $>95 \%$ pure cell populations. Fibroblasts were isolated from breast reduction mammoplasty tissue [47] and retrovirally transduced with pBABE-neo-hTERT [48] to increase longevity. Quarterly mycoplasma tests were consistently negative. With the exception of fibroblasts isolated inhouse, all cells were Short Tandem Repeat profiled annually by the Leeds Institutes of Molecular Medicine Genomics facility confirming no cross-contamination (last tested April 2014).

\section{Western blotting}

This was performed as previously [47] using NuPAGE® Novex ${ }^{\circledR} 4-12 \%$ Bis-Tris Gels (Invitrogen, Carlsbad, CA, USA) and proteins transferred to polyvinylidene difluoride membrane (Amersham Biosciences, Buckinghamshire, UK). Membranes were incubated $\left(4^{\circ} \mathrm{C}\right.$ overnight) with HER2 IgG (Rabbit Polyclonal, Cell Signalling Technology, dilution 1:1000), HER3 IgG (Mouse monoclonal, Clone 2F12, Millipore, dilution $1: 1000$ ) or $\beta$-actin IgG (Clone AC-15, Sigma, dilution 1:10000) and HRP conjugated secondary antibodies (Santa Cruz Biotechnology, Santa Cruz, USA; dilution 1:10000; $1 \mathrm{hr}$ ) before visualisation with SuperSignal West Pico Chemiluminescent Substrate (Thermo Scientific, Loughborough, UK).

\section{D collagen gel culture}

Myo1089 cells and fibroblasts were fluorescently labelled with pGIPZ-tGFP and pFURW-dsRed lentiviral vectors respectively and generated using a $2^{\text {nd }}$ generation lentiviral packaging system [49] to enable tracking in culture (Suppl. Figure 1) using immunohistochemistry. Rat Tail Collagen I (BD Biosciences, USA) was diluted with Dulbecco's Phospho Buffered Saline (DPBS, Invitrogen, Carlsbad, CA, USA; $2 \mathrm{mg} / \mathrm{mL}$ [2] and mixed with eight parts of collagen solution $(2 \mathrm{mg} / \mathrm{mL})$ to one part 10x Hanks Buffered Salt Solution (HBSS, Sigma, Poole, UK)). The gel was neutralised by drop-wise addition of $\mathrm{NaOH}$ until a pink-orange colour was achieved. 3D gel culture medium was prepared (50\% complete HAMF 12 Nutrient Mixture (Sigma, Poole, UK); 50\% complete Dulbecco's modified Eagle's medium (DMEM, Invitrogen, Carlsbad, CA, USA)). Myo1089, HB2 and fibroblast cells were mixed (6:2:1, respectively) in one volume of the above media to achieve a cell density of $4 \times 10^{5}$ cells $/ \mathrm{mL}$ gel. This was determined empirically and the ratio which best represented normal breast tissue phenotype (judged by $\mathrm{AMH}$, a specialist breast histopathologist), was selected. $200 \mu$ l of the gel-cell mixture was added to 12 -well plate Transwell ${ }^{\circledR}$ cell culture inserts with PET membranes and $8.0 \mu \mathrm{m}$ pore size (BD Falcon ${ }^{\circledR}$, USA) and placed within wells of a culture plate (Costar, Corning Inc, Corning, USA). Gels were allowed to solidify $\left(20 \mathrm{mins} 37^{\circ} \mathrm{C}\right.$ in $5 \%$ $\mathrm{CO}_{2}$ ) before adding $500 \mu 1$ of media to the top of each gel and $1 \mathrm{~mL}$ to the bottom of each well in the cell culture plate. Gels were cultured for 21 days with $50 \%$ of the media being replaced every three days during this time. Gels were fixed in $10 \%$ formalin (Sigma, Poole, UK) for 5 hours, processed (Leica ASP 200 tissue processor), paraffin embedded and $5 \mu \mathrm{m}$ sections taken using a Leica RM2235 microtome (both Leica Microsystems, Milton Keynes, UK) and mounted onto Superfrost Plus slides (BDH, Poole, UK). All slides were H\&E stained allowing visualisation.

\section{Immunohistochemistry}

This was performed on $5 \mu \mathrm{m}$ sections from reduction mammoplasty tissue and 3D gels. Primary antibodies included: vimentin (Clone Vim 3B4, 1:200), epithelial membrane antigen (EMA; Clone E29, 1:5000), $\alpha$-smooth muscle actin ( $\alpha$ SMA; Clone 1A4, 1:200), E-cadherin (Clone NCH-38, 1:100), Ki67 (Clone MIB1, 1:1000) [all Dako, Glostrup, Denmark], collagen IV (Clone COL94, 1:100, Sigma, Poole, UK], M30 (mouse anti-human monoclonal, Clone M30, 1:50, Roche Diagnostics, West Sussex, UK), turboGFP (rabbit polyclonal, 1:500, Pierce, Northumberland, UK) and dsRed (rabbit polyclonal, 1:200, Clontech Laboratories Inc, CA, USA). Primary antibody omission served as negative controls in each run. Antibodies were incubated overnight at $4^{\circ} \mathrm{C}$ with the exception of turboGFP and dsRed ( 2 hours, room temperature). Heat-induced antigen retrieval was used with the exception of those incubated with collagen IV which required proteolytic antigen retrieval. Here, sections were incubated at $37^{\circ} \mathrm{C}$ for $20 \mathrm{mins}$ in $20 \mu \mathrm{g} / \mathrm{mL}$ Proteinase $\mathrm{K}$ (Fungal, Invitrogen, Carlsbad, CA, USA) in Phosphate Buffered Saline (PBS) prior to blocking. An extra blocking step was used prior to antibody incubation; sections were rinsed twice with $2 \%$ Bovine Serum Albumin (BSA, Sigma, Poole, UK) in PBS before adding $100 \mu 120 \%$ goat serum (Dako, Glostrup, Denmark) in PBS (incubation at room temperature for 20 mins).

\section{Image acquisition}

Slides were scanned (Aperio CS and AT scanners; Aperio Technologies Inc, Vista, CA, USA) using x20 and $\mathrm{x} 40$ objectives producing images with a final resolution of 0.23 and $0.43 \mu \mathrm{m}$ per pixel respectively. Digital images were acquired using Aperio Imagescope software version 11. 


\section{Quantification of 3D in vitro tri-culture model}

Annotation tools were used to outline the perimeters of the whole gel section from digital images at 20x magnification, any holes within the gel section and around HB2 units were excluded manually using a computer equipped with a Wacom Cintiq touchscreen monitor. A viable HB2 unit was defined as a cohesive cluster of four or more cells with discernible nuclei. To ensure that units quantified were comprised of HB2 cells, IHC was performed alongside $\mathrm{H} \& \mathrm{E}$ of the gels to confirm that the structures being annotated did not contain turboGFP or dsRed positive cells. HB2 units on the periphery of the gel section or holes were excluded as they did not represent the majority of HB2 structures within the gels (Suppl. Figure 2). Aperio Imagescope software calculated the number of annotations drawn and area of each annotation in $\mu \mathrm{m}^{2}$. Lumen formation was defined as a space within HB2 units that was enclosed by a continuous chain of cells and was counted manually. Any HB2 units that contained luminal spaces with breaches in the surrounding cells were excluded (Suppl. Figure 2). The area of the gel section was calculated by subtracting the area of holes in the gel from the total area of the gel section. Due to the inherent variation between the size of gel sections and therefore the number of units within each gel, the area of HB2 units were normalised by expressing the area of the unit per $\mu \mathrm{m}^{2}$ of gel. This was calculated by taking the area of each HB2 unit and dividing by the area of the gel section. For each experiment, one section from three replicate gels for each variable was quantified. Raw data was inputted into GraphPad Prism 6 software where the mean and SEM was calculated along with statistical analysis. For comparison of the area, number and lumen formation, unpaired t-test analysis was performed where $p<0.05$ was considered significant.

\section{ACKNOWLEDGEMENTS}

CEN was supported by a Leeds Institute of Molecular Medicine PhD studentship. VS, AMH and FB received grants from Breast Cancer Campaign, which supported DLH and EWB. We acknowledge the support of the Breast Cancer Campaign Tissue Bank in the generation of fibroblasts used in this work.

\section{$\begin{array}{lll}\text { STATEMENT OF AUTHOR } & \text { OF }\end{array}$ CONTRIBUTIONS}

CEN, VS, GM, DLH, DCT, DT and AMH designed the experiments. CEN conducted all of the experiments. EWB generated immortalised fibroblasts. FB and VN generated HER overexpressing cells. CEN, VS, GM, DCT and AMH analysed the data. All authors contributed to the manuscript preparation.

\section{CONFLICTS OF INTEREST}

The authors declare they have no conflicts of interest

\section{REFERENCES}

1. Dhimolea E, Maffini MV, Soto AM and Sonnenschein C. The role of collagen reorganization on mammary epithelial morphogenesis in a 3D culture model. Biomaterials. 2010; 31:3622-3630.

2. Holliday DL, Brouilette KT, Markert A, Gordon LA and Jones JL. Novel multicellular organotypic models of normal and malignant breast: tools for dissecting the role of the microenvironment in breast cancer progression. Breast Cancer Research. 2009; 11:R3.

3. Krause S, Maffini MV, Soto AM and Sonnenschein C. A Novel 3D In Vitro Culture Model to Study StromalEpithelial Interactions in the Mammary Gland. Tissue Engineering Part C: Methods. 2008; 14:261-271.

4. Streuli CH, Bailey N and Bissell MJ. Control of mammary epithelial differentiation: basement membrane induces tissue-specific gene expression in the absence of cell-cell interaction and morphological polarity. The Journal of Cell Biology. 1991; 115:1383-1395.

5. Lee GY, Kenny PA, Lee EH and Bissell MJ. Threedimensional culture models of normal and malignant breast epithelial cells. Nat Methods. 2007; 4:359-365.

6. Weaver VM, Petersen OW, Wang F, Larabell CA, Briand $\mathrm{P}$, Damsky C and Bissell MJ. Reversion of the Malignant Phenotype of Human Breast Cells in Three-Dimensional Culture and In Vivo by Integrin Blocking Antibodies. The Journal of Cell Biology. 1997; 137:231-245.

7. Runswick SK, O'Hare MJ, Jones L, Streuli CH and Garrod DR. Desmosomal adhesion regulates epithelial morphogenesis and cell positioning. Nat Cell Biol. 2001; 3:823-830.

8. Holliday D and Speirs V. Choosing the right cell line for breast cancer research. Breast Cancer Research. 2011; 13(4):215

9. Debnath J, Muthuswamy SK and Brugge JS. Morphogenesis and oncogenesis of MCF-10A mammary epithelial acini grown in three-dimensional basement membrane cultures. Methods. 2003; 30:256-268.

10. Petersen OW, Ronnov-Jessen L, Howlett AR and Bissell MJ. Interaction with basement membrane serves to rapidly distinguish growth and differentiation pattern of normal and malignant human breast epithelial cells. Proc Natl Acad Sci U S A. 1992; 89:9064-9068.

11. DiRenzo J, Signoretti S, Nakamura N, Rivera-Gonzalez R, Sellers W, Loda M and Brown M. Growth factor requirements and basal phenotype of an immortalized mammary epithelial cell line. Cancer research. 2002; 62:8998.

12. Soule HD, Maloney TM, Wolman SR, Peterson WD, 
Jr., Brenz R, McGrath CM, Russo J, Pauley RJ, Jones $\mathrm{RF}$ and Brooks SC. Isolation and characterization of a spontaneously immortalized human breast epithelial cell line, MCF-10. Cancer research. 1990; 50:6075-6086.

13. Yusuf R and Frenkel K. Morphologic transformation of human breast epithelial cells MCF-10A: dependence on an oxidative microenvironment and estrogen/epidermal growth factor receptors. Cancer Cell International. 2010; 10:30.

14. Wisdom BJ, Gunwar S, Hudson MD, Noelken ME and Hudson BG. Type IV Collagen of Engelbreth-HolmSwarm Tumor Matrix: Identification of Constituent Chains. Connective Tissue Research. 1992; 27:225-234.

15. Parmar $\mathrm{H}$ and Cunha GR. Epithelial-stromal interactions in the mouse and human mammary gland in vivo. Endocr Relat Cancer. 2004; 11:437-458.

16. Provenzano P, Eliceiri K, Campbell J, Inman D, White J and Keely P. Collagen reorganization at the tumor-stromal interface facilitates local invasion. BMC Medicine. 2006; 4:38.

17. Provenzano PP, Inman DR, Eliceiri KW and Keely PJ. Matrix density-induced mechanoregulation of breast cell phenotype, signaling and gene expression through a FAKERK linkage. Oncogene. 2009; 28:4326-4343.

18. Maskarinec G, Pagano IS, Little MA, Conroy SM, Park SY and Kolonel LN. Mammographic density as a predictor of breast cancer survival: the Multiethnic Cohort. Breast Cancer Res. 2013; 15(1).

19. Alowami S, Troup S, Al-Haddad S, Kirkpatrick I and Watson P. Mammographic density is related to stroma and stromal proteoglycan expression. Breast Cancer Res. 2003; 5:R129 - R135.

20. Kojima Y, Acar A, Eaton EN, Mellody KT, Scheel C, BenPorath I, Onder TT, Wang ZC, Richardson AL, Weinberg RA and Orimo A. Autocrine TGF-beta and stromal cellderived factor-1 (SDF-1) signaling drives the evolution of tumor-promoting mammary stromal myofibroblasts. Proc Natl Acad Sci U S A. 2010; 107:20009-20014.

21. Olsen C, Moreira J, Lukanidin E and Ambartsumian N. Human mammary fibroblasts stimulate invasion of breast cancer cells in a three-dimensional culture and increase stroma development in mouse xenografts. BMC Cancer. 2010; 10:444.

22. Tyan SW, Kuo WH, Huang CK, Pan CC, Shew JY, Chang $\mathrm{KJ}$, Lee EY and Lee WH. Breast cancer cells induce cancerassociated fibroblasts to secrete hepatocyte growth factor to enhance breast tumorigenesis. PloS one. 2011; 6:0015313.

23. Runswick SK, O’Hare MJ, Jones L, Streuli CH and Garrod DR. Desmosomal adhesion regulates epithelial morphogenesis and cell positioning. Nat Cell Biol. 2001; 3:823-830.

24. Gudjonsson T, Ronnov-Jessen L, Villadsen R, Rank F, Bissell MJ and Petersen OW. Normal and tumor-derived myoepithelial cells differ in their ability to interact with luminal breast epithelial cells for polarity and basement membrane deposition. J Cell Sci. 2002; 115:39-50.

25. Barsky SH. Myoepithelial mRNA expression profiling reveals a common tumor-suppressor phenotype. Experimental and Molecular Pathology. 2003; 74:113-122.

26. Jones JL, Shaw JA, Pringle JH and Walker RA. Primary breast myopithelial cells exert an invasion-suppressor effect on breast cancer cells via paracrine down-regulation of MMP expresssion in fibroblasts and tumour cells. Journal of Pathology. 2003; 201:562-572.

27. Sternlicht MD, Safarians S, Rivera SP and Barsky SH. Characterizations of the extracellular matrix and proteinase inhibitor content of human myoepithelial tumors. Lab Invest. 1996; 74:781-796.

28. Speirs V, Skliris GP, Burdall SE and Carder PJ. Distinct expression patterns of ER $\alpha$ and $E R \beta$ in normal human mammary gland. Journal of Clinical Pathology. 2002; 55:371-374.

29. Berdichevsky F, Alford D, D'Souza B and TaylorPapadimitriou J. Branching morphogenesis of human mammary epithelial cells in collagen gels. Journal of Cell Science. 1994; 107:3557-3568.

30. Novitskaya V, Romanska H, Dawoud M, Jones JL and Berditchevski F. Tetraspanin CD151 regulates growth of mammary epithelial cells in three-dimensional extracellular matrix: implication for mammary ductal carcinoma in situ. Cancer research. 2010; 70:4698-4708.

31. Choritz H, Busche G and Kreipe H. Quality assessment of HER2 testing by monitoring of positivity rates. Virchows Arch. 2011; 459:283-289.

32. Slamon DJ, Clark GM, Wong SG, Levin WJ, Ullrich A and McGuire WL. Human breast cancer: correlation of relapse and survival with amplification of the HER-2/neu oncogene. Science. 1987; 235:177-182.

33. Park K, Han S, Kim HJ, Kim J and Shin E. HER2 status in pure ductal carcinoma in situ and in the intraductal and invasive components of invasive ductal carcinoma determined by fluorescence in situ hybridization and immunohistochemistry. Histopathology. 2006; 48:702-707.

34. Guy CT, Cardiff RD and Muller WJ. Activated neu Induces Rapid Tumor Progression. Journal of Biological Chemistry. 1996; 271:7673-7678.

35. Muller WJ, Sinn E, Pattengale PK, Wallace R and Leder P. Single-step induction of mammary adenocarcinoma in transgenic mice bearing the activated c-neu oncogene. Cell. 1988; 54:105-115.

36. Hellyer NJ, Cheng K and Koland JG. ErbB3 (HER3) interaction with the $\mathrm{p} 85$ regulatory subunit of phosphoinositide 3-kinase. Biochem J. 1998; 333:757-763.

37. Kurokawa H, Lenferink AE, Simpson JF, Pisacane PI, Sliwkowski MX, Forbes JT and Arteaga CL. Inhibition of HER2/neu (erbB-2) and mitogen-activated protein kinases enhances tamoxifen action against HER2-overexpressing, tamoxifen-resistant breast cancer cells. Cancer research. 2000; 60:5887-5894. 
38. Shou J, Massarweh S, Osborne CK, Wakeling AE, Ali $\mathrm{S}$, Weiss $\mathrm{H}$ and Schiff R. Mechanisms of Tamoxifen Resistance: Increased Estrogen Receptor-HER2/neu CrossTalk in ER/HER2-Positive Breast Cancer. Journal of the National Cancer Institute. 2004; 96:926-935.

39. Ozbay $T$ and Nahta R. Delphinidin Inhibits HER2 and Erk1/2 Signaling and Suppresses Growth of HER2Overexpressing and Triple Negative Breast Cancer Cell Lines. Breast Cancer (Auckl). 2011; 5:143-154.

40. Pradeep CR, Zeisel A, Kostler WJ, Lauriola M, JacobHirsch J, Haibe-Kains B, Amariglio N, Ben-Chetrit N, Emde A, Solomonov I, Neufeld G, Piccart M, Sagi I, Sotiriou C, Rechavi G, Domany E, et al. Modeling invasive breast cancer: growth factors propel progression of HER2positive premalignant lesions. Oncogene. 2012; 31:35693583.

41. Aceto N, Duss S, MacDonald G, Meyer D, Roloff T-C, Hynes N and Bentires-Alj M. Co-expression of HER2 and HER3 receptor tyrosine kinases enhances invasion of breast cells via stimulation of interleukin-8 autocrine secretion. Breast Cancer Research. 2012; 14:R131.

42. Dittmar T, Husemann A, Schewe Y, Nofer J-R, Niggemann $\mathrm{B}$, Zänker KS and Brandt BH. Induction of cancer cell migration by epidermal growth factor is initiated by specific phosphorylation of tyrosine 1248 of c-erbB-2 receptor via epidermal growth factor receptor. The FASEB Journal. 2002.

43. Wolf-Yadlin A, Kumar N, Zhang Y, Hautaniemi S, Zaman M, Kim HD, Grantcharova V, Lauffenburger DA and White FM. Effects of HER2 overexpression on cell signaling networks governing proliferation and migration. Mol Syst Biol. 2006; 2:3.

44. Bartek J, Bartkova J, Kyprianou N, Lalani EN, Staskova Z, Shearer M, Chang S and Taylor-Papadimitriou J. Efficient immortalization of luminal epithelial cells from human mammary gland by introduction of simian virus 40 large tumor antigen with a recombinant retrovirus. Proc Natl Acad Sci U S A 1991. 88:3520-3524.

45. D'Souza B, Berdichevsky F, Kyprianou N and TaylorPapadimitriou J. Collagen-induced morphogenesis and expression of the alpha 2-integrin subunit is inhibited in c-erbB2-transfected human mammary epithelial cells. Oncogene. 1993; 8:1797-1806.

46. Riese DJ, 2nd, van Raaij TM, Plowman GD, Andrews GC and Stern DF. The cellular response to neuregulins is governed by complex interactions of the erbB receptor family. Molecular and cellular biology. 1995; 15:57705776.

47. Simpkins SA, Hanby AM, Holliday DL and Speirs V. Clinical and functional significance of loss of caveolin-1 expression in breast cancer-associated fibroblasts. The Journal of pathology. 2012; 227:490-498.

48. Tomlinson DC, Baxter EW, Loadman PM, Hull MA and Knowles MA. FGFR1-induced epithelial to mesenchymal transition through MAPK/PLCgamma/COX-2-mediated mechanisms. PloS one. 2012; 7:e38972.

49. EPFL. (2012). Lentivectors Toolbox. 